\title{
Changes in Inflammatory Cytokines and Irisin in Response to High Intensity Swimming in Adolescent versus Adult Male Swimmers
}

\author{
Malcolm Sanderson ${ }^{1}$, Brandon J. McKinlay ${ }^{1,2}$, Alexandros Theocharidis ${ }^{1}$, Rozalia Kouvelioti ${ }^{1,2}$, \\ Bareket Falk 1,2 (D) and Panagiota Klentrou 1,2,*D \\ 1 Department of Kinesiology, Brock University, St. Catharines, ON L2S 3A1, Canada; \\ ms16ot@brocku.ca (M.S.); brandon.mckinlay@brocku.ca (B.J.M.); theocharidisalex@gmail.com (A.T.); \\ rkouvelioti@brocku.ca (R.K.); bfalk@brocku.ca (B.F.) \\ 2 Centre for Bone and Muscle Health, Brock University, St. Catharines, ON L2S 3A1, Canada \\ * Correspondence: nklentrou@brocku.ca
}

Received: 30 October 2020; Accepted: 26 November 2020; Published: 1 December 2020

\begin{abstract}
Swimming is a popular youth sport that is considered beneficial for cardiovascular fitness. However, the potential inflammatory outcomes of high intensity swimming in younger swimmers are unclear, as is the response of irisin, a myokine released during exercise with anti-inflammatory properties. This study compared the plasma concentrations of interleukins 1-beta (IL-1 $\beta$ ), 6 (IL-6), 10 (IL-10), tumor necrosis factor alpha (TNF- $\alpha$ ) and irisin in response to intense swimming between adolescent and adult male swimmers. Thirty-two swimmers (16 adolescents, $14 \pm 1$ years; 16 adults, $21.5 \pm 3.1$ years) completed a high intensity interval swimming trial. At rest, only TNF- $\alpha$ was higher $(33 \%, p<0.05)$ in adolescents compared with adults. There was an overall significant increase in IL-1 $\beta$ from pre- to post-swimming ( $3 \%$ in adolescents, $24 \%$ in adults), but no significant interaction. IL-10 significantly increased in both groups ( $+34 \%$ in adolescents, $+56 \%$ in adults). IL- 6 and TNF- $\alpha$ increased significantly ( $+32 \%$ and $+26 \%$, respectively) in adults, but not in adolescents $(+2 \%$ and $-9 \%$, respectively). Adults showed a small, but significant decrease in irisin $(-5 \%)$, with no change in adolescents. The lack of an IL- 6 , TNF- $\alpha$ and irisin response to intense swimming in adolescent swimmers may suggest a blunted inflammatory and myokine response following high intensity exercise in trained youth.
\end{abstract}

Keywords: boys; men; athletes; high-intensity interval exercise; inflammation

\section{Introduction}

Intense exercise can significantly influence several inflammatory markers, while the magnitude and direction of changes in specific cytokines, may be mediated by the mode and intensity of the activity, as well as the age and training status of participants [1,2]. For example, although children recover faster than adults from intense exercise, not just in terms of performance, but also in terms of physiological responses [3,4], there is no consistent evidence of age-related differences in the inflammatory response to intense exercise. In recent years, there has been increasing interest in studying the inflammatory response after various forms of intense exercise in both adults and children involved in high performance sports, due to the potential that repetitive bouts of high intensity exercise with little recovery time may lead to chronic low-grade inflammation [1].

Interleukin 1-beta (IL-1 $\beta$ ), interleukin 6 (IL-6), interleukin 10 (IL-10), and tumor necrosis factor alpha (TNF- $\alpha)$ are known pro-inflammatory cytokines that are released in the early stages of the inflammation process in response to muscle injury to initiate the muscle regeneration process [5]. IL-10 is an anti-inflammatory cytokine released later to repress the pro-inflammatory effects and 
to support potential differentiation of muscle stem cells [5]. In young, non-athletic men, previous research has observed increases in TNF- $\alpha$ [6-8], IL-6 [6-9], and IL-10 [6-8], but not IL-1 $\beta$ [8], following various exercise protocols compared to pre-exercise levels. However, there has been much less research regarding the cytokine response to exercise in children and adolescents, with studies reporting mixed results of both increases [10-12], and no changes post-exercise [9]. Although the intensity of these studies ranges from moderate to intense, and irrespective of the limited evidence, there is agreement that muscle damage associated with exercise, which produces an inflammatory response, may be less in children than in adults $[13,14]$. However, whether this blunted inflammatory response is also true for youth athletes is unclear. In addition, irisin is a myokine secreted by skeletal muscles during exercise, which induces browning of white adipose tissue [15]. Recent evidence also suggests that irisin may also have anti-inflammatory properties, leading to the reduction of secretion of inflammatory cytokines like IL-6 and TNF- $\alpha$ [16]. Irisin has been shown to immediately increase post-exercise in both adults [17-20] and children [20,21] while its response to exercise is intensity-dependent, with larger post-exercise increases following higher intensity exercise [17].

Most available data on the inflammatory response to exercise relate to prolonged, moderate exercise. However, the increase in cytokine levels following repeated bouts of intense exercise, may be attenuated compared with the response following the initial bout [22]. It is possible that the anti-inflammatory cytokine IL-10 and irisin are related to the attenuated response following repeated bouts. In addition, there are no studies that have directly compared the acute post-exercise differences in cytokine and irisin responses to the same intense exercise protocol in adolescent and adult athletes. Swimming, which is associated with metabolic, musculoskeletal, and cardiorespiratory benefits [23], is a popular sport among youth. It is, however, different from other land-based sports, in that is predominantly concentric and low impact, and has been shown to cause different biochemical reactions than land-based sports [24]. Indeed, swimming generally induces less muscle damage, and potentially a lower inflammatory response, compared to eccentric activities (e.g., resistance or plyometric exercise), which are known to result in considerable inflammation, especially during novel exercise [25]. Thus, the purpose of this study was to examine the age-related differences in the acute response of antiand pro-inflammatory cytokines (IL-1 $\beta$, IL- 6 , IL-10, and TNF- $\alpha$ ) and irisin to a high intensity interval swimming trial in adolescent and adult male swimmers.

\section{Materials and Methods}

\subsection{Participants}

Participants in the current study included 16 adolescent (11-15 years old) and 16 adult (18-30 years old) male swimmers, who trained and competed for at least four years, were free of injuries and any medical condition that prevented them from participating and were not taking medications or nutritional supplements. Swimmers were recruited from two varsity teams and five local swim clubs in Southern Ontario as part of a larger study designed to examine the effect of post-exercise whey protein consumption on subsequent exercise performance, muscle damage, and inflammation [26]. Data presented in this study are a secondary analyses of blood samples taken before the protein/carbohydrate supplementation protocol and solely focus on acute responses to intense swimming.

All participants and their parents/guardians were provided a thorough explanation of the study's purpose, procedures, and potential risks. Consent was obtained from all participants and their respective parent/guardian where applicable. The study procedures and secondary analysis presented herein were cleared by the Research Ethics Boards of Brock University (REB\# 16-279 and 18-296, respectively) and the Canadian Sports Institute of Ontario (REB\# 2017-01). 


\subsection{Study Design}

The study employed a parallel cross-sectional design which included two visits. The first visit was an information session, which also included anthropometric measurements. Details of the measurements can be found in McKinlay et al. [26]. In brief, body fat and body mass (kg) were collected using bioelectric impedance analysis (InBody520; BioSpace Co, Ltd., Madison, MI, USA). Participants self-hydrated ad libitum prior to the visit and were asked to void before body measurements were performed. Seated and standing height were recorded with a stadiometer (SECA-217; CAN), and were used to assess somatic maturity, indicated as years from age of peak height velocity [27].

Between visits 1 and 2, participants were instructed to refrain from any exercise for a minimum of $48 \mathrm{~h}$ prior to visit 2 . The second visit included the swimming trial and two blood samples: one fasted (i.e., after an overnight fast of 10-12 h), a resting (i.e., pre-swimming) sample collected at 06:00 h, and one post-swimming sample collected within $15 \mathrm{~min}$ following swimming. Both the swimming trial and the blood draws are described below. Immediately after the resting blood sample and $\sim 1 \mathrm{~h}$ before swimming, participants were provided a standardized breakfast of $\sim 300-500 \mathrm{kcal}$ (depending on body mass), which included a granola bar, muffin, fruit (banana or apple) and a juice box or water. Details of the ingredients of the breakfast can be found in McKinlay et al. [26].

\subsection{High Intensity Swimming Trial}

The swimming trial commenced $\sim 1.5 \mathrm{~h}$ after the pre-exercise blood draw ( $\sim \mathrm{h}$ postprandial) and it was designed for the purposes of the larger study to mirror a day of swimming competition. Details of the larger study's protocol and measurements can be found in McKinlay et al. [26]. Briefly, the trial started with participants performing a warm-up of a $1000 \mathrm{~m}$ swim, followed by a maximal $200 \mathrm{~m}$ front-crawl swim test. Approximately $5 \mathrm{~min}$ following the maximal swim test, participants performed a high intensity interval swimming (HIIS) protocol, which consisted of $5 \times 100 \mathrm{~m}, 5 \times 50 \mathrm{~m}$ and $5 \times 25 \mathrm{~m}$ at $>90 \%$ of each swimmer's maximum performance time, as calculated from their split time $(100 \mathrm{~m})$ during the $200 \mathrm{~m}$ maximal swim test, and using a 1:1 work-to-rest-ratio.

\subsection{Blood Collection and Analysis}

In order to control for circadian rhythm, all testing was performed in the morning hours. Blood samples were collected at rest (06:00 h, fasted), and immediately (within $15 \mathrm{~min}$ ) following the swimming trial (09:00-10:00 h). Venous samples $(10 \mathrm{~mL})$ were drawn from the antecubital fossa using a standard venipuncture technique. All samples were centrifuged at $1405 \times g$ at $4{ }^{\circ} \mathrm{C}$ using a benchtop centrifuge for $10 \mathrm{~min}$. Serum and plasma were then aliquoted into pre-labeled Eppendorf tubes to be stored at $-80^{\circ} \mathrm{C}$ until analysis.

Plasma concentrations of IL-1 $\beta$, IL-6, IL-10 and TNF- $\alpha$ were measured in duplicates using multiplex magnetic bead kits (Cat. \#HSTCMAG-28SK, Milliplex EMD Millipore Corporation, Burlington, MA, USA). The average in-house inter- and intra-assay coefficients of variation (CV) for IL-6, IL-10, IL-1 $\beta$ and TNF- $\alpha$ were $8.1 \%$ and $4.3 \%, 6.5 \%$ and $4.3 \%, 4.6 \%$ and $7.3 \%, 8 \%$ and $5.3 \%$, respectively. Irisin was also measured in duplicate using an irisin recombinant assay kit (Cat \#EK-067-29, Phoenix Pharmaceuticals Inc., Burlingame, CA, USA). The average in-house inter- and intra-assay CV for irisin were $1.2 \%$ and $4 \%$, respectively.

For every blood draw, hematocrit was measured in triplicates by the same investigator using microhematocrit capillary tubes treated with heparin (VWR International, Radnor, PA, USA). Relative plasma volume changes $(\% \triangle P V)$ were calculated using the formula by Van Beaumont [28].

$$
\% \Delta P V=\frac{100}{100-H c t 1} \times \frac{100(H c t 1-H c t 2)}{H c t 2} \%
$$


where Hct1 is hematocrit at baseline, and Hct2 is hematocrit at each post-baseline measurement. The $\% \triangle P V$ was then used to adjust the post-swimming plasma concentrations of inflammatory cytokines (IL-6, IL-10, IL-1 $\beta$, TNF- $\alpha$ ) and irisin using the formula: $100+\% \Delta P V / 100$.

\subsection{Statistical Analysis}

All variables were first checked for normality using the Kolmogorov-Smirnov test; variables were also checked for skewness and kurtosis of \pm 3 . In addition, visual screening of histograms for symmetry was performed. IL-1 $\beta$, IL-6, IL-10 and irisin concentrations were not normally distributed, and were log transformed for further analysis. Group differences in physical characteristics, resting cytokines and irisin levels were analyzed using independent t-tests. A two-way (group-by-time) ANOVA for repeated measures was used to examine changes in cytokines (IL-1 $\beta$, IL-6, IL-10, TNF- $\alpha$ ) and irisin levels (pre- to post-swimming). In the event of a significant interaction, post-hoc comparisons were performed within each group using paired t-tests. The baseline characteristics are reported as mean \pm standard deviation (SD) and cytokine concentrations at rest are reported as mean \pm standard error (SE) in descriptive tables. The results of the ANOVA repeated measurements are shown reporting F, $p$ and partial $\eta^{2}$. Effect size for partial $\eta^{2}$ was then interpreted based on the Cohen criteria: $0.01=$ small effect, $0.06=$ moderate effect, and $0.14=$ large effect. Statistical significance was accepted at $p<0.05$, which was conducted using SPSS version 26 for Windows (SPSS Inc., Chicago, IL, USA).

\section{Results}

Baseline physical and training characteristics can be found in Table 1 and baseline (i.e., resting) concentrations of cytokines and irisin are presented in Table 2. Inflammatory cytokine levels at rest were not significantly different between groups, with the exception of TNF- $\alpha$, which was $+33 \%$ higher $(p=0.02)$ in the adolescent male swimmers compared to the adults (Table 2).

Table 1. Participant physical and training characteristics (values are mean \pm standard deviation).

\begin{tabular}{ccc}
\hline Variable & Adolescent Swimmers $(\boldsymbol{n = 1 6 )}$ & Adult Swimmers $(\boldsymbol{n}=\mathbf{1 6})$ \\
\hline Age $(\mathrm{y})$ & $14.0 \pm 1.0$ & $21.5 \pm 3.1^{*}$ \\
Somatic maturity & $0.6 \pm 1.0$ & $\mathrm{n} / \mathrm{a}$ \\
(y from age of peak height velocity) & $170.0 \pm 9.3$ & $179.6 \pm 7.4^{*}$ \\
Height (cm) & $56.5 \pm 10.5$ & $76.7 \pm 10.7^{*}$ \\
Mass $(\mathrm{kg})$ & $11.7 \pm 3.7$ & $14.1 \pm 5.2$ \\
Body Fat $(\%)$ & $4.7 \pm 1.3$ & $9.1 \pm 3.7^{*}$ \\
Training History (y) & $13.7 \pm 0.9$ & $15.0 \pm 1.3$ \\
Training Volume (h/wk) &
\end{tabular}

* denotes a significant difference between groups $(p<0.05)$.

Figure 1 shows the percent changes in the various cytokines from pre- to post-swimming in each group. IL- $1 \beta$ showed a significant effect for time $\left(\mathrm{F}=6.47 ; p=0.016\right.$; partial $\left.\eta^{2}=0.18\right)$, reflecting an overall large increase from pre to post-exercise, with no significant group effect $(\mathrm{F}=0.00 ; p=0.99$; partial $\left.\eta^{2}=0.00\right)$ and no significant group-by-time interaction $\left(\mathrm{F}=2.87 ; p=0.10\right.$; partial $\left.\eta^{2}=0.09\right)$. Thus, although IL- $1 \beta$ increased by $3 \%$ in adolescents versus $24 \%$ in adults, this difference was not statistically significant (Figure 1). IL-6 showed no significant group effect $\left(\mathrm{F}=1.14 ; p=0.29\right.$; partial $\left.\eta^{2}=0.03\right)$, but a significant time effect $\left(\mathrm{F}=14.11 ; p=0.001\right.$; partial $\left.\eta^{2}=0.32\right)$ and a large, significant group-by-time interaction $\left(\mathrm{F}=9.75 ; p=0.004\right.$; partial $\left.\eta^{2}=0.25\right)$, reflecting a significant increase following intense swimming in the adult swimmers $(+32 \%, p=0.002)$, which was not the case in adolescents (Figure 1$)$. There was a large, significant increase over time for IL- 10 ( $+34 \%$ and $+56 \%$ in boys and men, respectively; $\mathrm{F}=40.6 ; p=0.001$; partial $\left.\eta^{2}=0.57\right)$, with no significant group effect $\left(\mathrm{F}=0.49 ; p=0.49\right.$; partial $\left.\eta^{2}=0.01\right)$ or group-by-time interaction $\left(\mathrm{F}=0.99 ; p=0.33\right.$; partial $\left.\eta^{2}=0.03\right)$. For TNF- $\alpha$, we found no effect for group $\left(\mathrm{F}=2.61 ; p=0.12 ;\right.$ partial $\left.\eta^{2}=0.08\right)$, a significant time effect $\left(\mathrm{F}=9.16 ; p=0.005 ;\right.$ partial $\left.\eta^{2}=0.23\right)$, and a significant group-by-time interaction $\left(\mathrm{F}=16.37 ; p=0.001 ;\right.$ partial $\left.\eta^{2}=0.35\right)$, which reflects a 
significant increase in the adults $(+26 \%, p<0.001)$ with a non-significant decrease $(-9 \%, p=0.47)$ in the adolescents (Figure 1). A significant main effect for time $\left(F=4.71 ; p=0.038\right.$; partial $\left.\eta^{2}=0.14\right)$, but not for the group $\left(\mathrm{F}=0.29 ; p=0.59\right.$; partial $\left.\eta^{2}=0.01\right)$, and a significant interaction $(\mathrm{F}=4.92 ; p=0.034$; partial $\left.\eta^{2}=0.14\right)$ were also found for irisin, reflecting a small yet significant decrease in adults $(-5 \%$, $p=0.03$ ), with no change in the adolescents (Figure 1).

Table 2. Resting (pre-swimming) circulating levels of inflammatory cytokines and myokines in adolescent and adult swimmers.

\begin{tabular}{cccc}
\hline Cytokine & Group & Mean \pm SE & Difference \\
\hline IL-1 $\beta(\mathrm{pg} / \mathrm{mL})$ & Adolescents & $9.81 \pm 5.47$ & $p=0.76$ \\
\hline & Adults & $8.32 \pm 6.27$ & \\
\hline $\mathrm{IL}-6(\mathrm{pg} / \mathrm{mL})$ & Adolescents & $28.29 \pm 9.60$ & $p=0.16$ \\
\hline & Adults & $14.94 \pm 6.70$ & $p=0.88$ \\
\hline $\mathrm{IL}-10(\mathrm{pg} / \mathrm{mL})$ & Adolescents & $12.62 \pm 1.61$ & \\
\hline & Adults & $14.79 \pm 3.82$ & \\
\hline TNF- $\alpha(\mathrm{pg} / \mathrm{mL})$ & Adolescents & $15.56 \pm 1.19$ & \\
\hline & Adults & $10.43 \pm 0.94$ & $p=0.45$ \\
\hline Irisin $(\mathrm{ng} / \mathrm{mL})$ & Adolescents & $13.23 \pm 0.97$ & \\
\hline & Adults & $14.49 \pm 1.31$ & \\
\hline
\end{tabular}

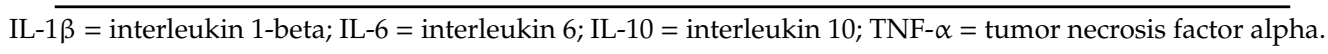

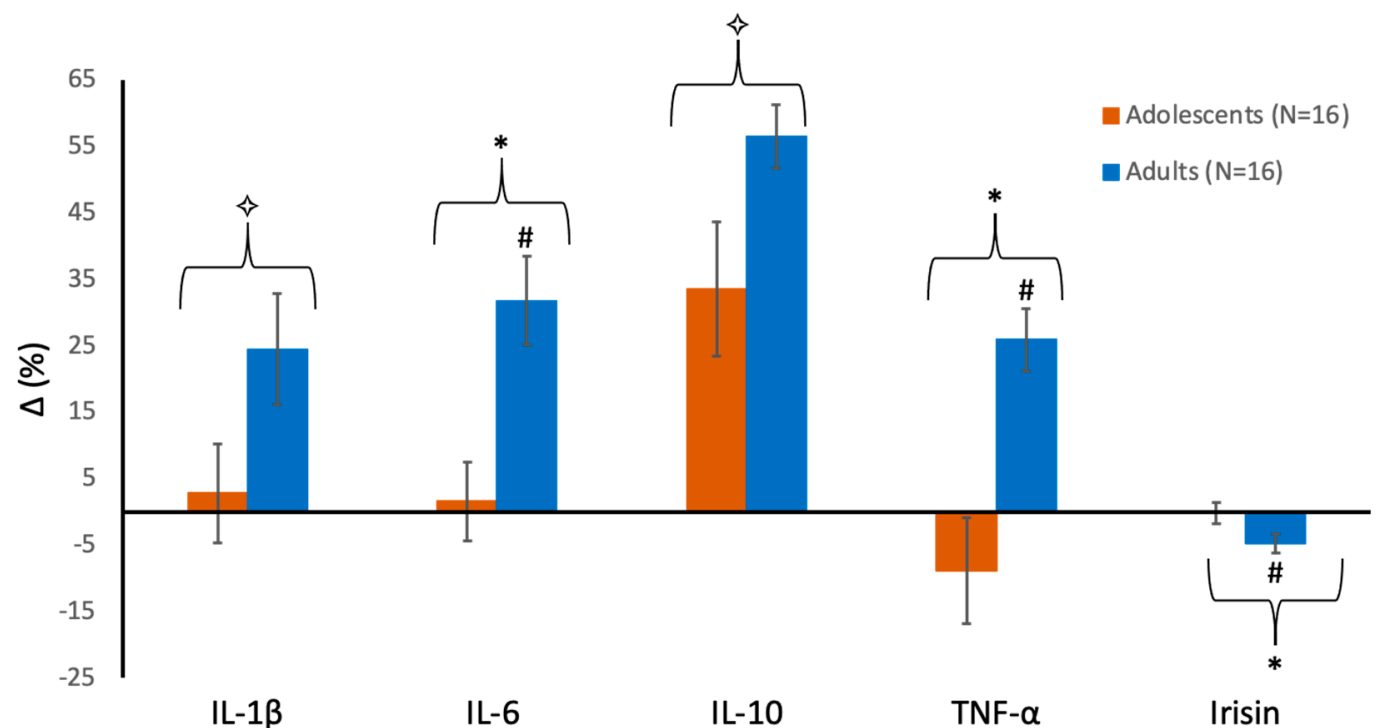

Figure 1. Percent changes ( $\Delta$ mean \pm standard error) from pre- to post-swimming in plasma concentrations of interleukin 1-beta (IL-1 $\beta$ ), interleukin 6 (IL-6), interleukin 10 (IL-10), tumor necrosis factor alpha (TNF- $\alpha$ ) and irisin in adolescent and adult male swimmers. * denotes a significant difference between groups (group effect, $p<0.05$ ). $\diamond$ denotes a significant change from pre- to post-swimming in both groups (time effect, $p<0.05$ ). \# denotes a significant change from pre- to post-swimming in adults (interaction, $p<0.05)$.

\section{Discussion}

To our knowledge, this is the first study to directly compare the response of anti- and pro-inflammatory cytokines, and irisin to high intensity interval swimming between two age groups of competitive male swimmers. Previous studies typically examined age-related differences in cytokine 
and irisin responses to acute exercise in non-athletic populations, who on one hand are accustomed to the specific exercise, but on the other hand are exposed to chronic high intensity, non-eccentric, exercise with little recovery time. Overall, we observed significant exercise-induced increases in all cytokines, and a decrease in irisin, in adults, but these changes were blunted or not evident in the adolescent swimmers, as evidenced by large interaction effects (partial $\eta^{2}$ ranging from 0.14 to 0.35 ). Specifically, IL-1 $\beta$, IL-6, IL-10 and TNF- $\alpha$ significantly increased while irisin decreased from preto post-swimming in the adults, but in the adolescents, only the anti-inflammatory IL-10 increased significantly in response to high intensity swimming.

At rest, IL-1 $\beta$, IL-10 and irisin levels were not significantly different between groups. The only significant age-related difference at rest was in TNF- $\alpha$, which was $33 \%$ higher in the adolescent group compared with adults. Notably, IL-6 was also $47 \%$ higher in the adolescents, but this difference was not statistically significant, likely due to its high variability. Studies examining age-related differences in the resting concentrations of inflammatory cytokines are scarce. Timmons et al. [9] also found significantly higher resting levels of IL-6, in their cohort of healthy, non-athletic boys ( $9.8 \pm 0.1$ years) compared to 10 young men of similar age to our adult swimmers ( $22.1 \pm 0.5$ years). They also found higher resting TNF- $\alpha$ in the boys, albeit the difference was not statistically different. It is possible that the higher resting TNF- $\alpha$ in adolescent swimmers could be indicative of low-grade chronic inflammation due to the high-volume training $(\sim 14 \mathrm{~h} /$ week $)$ in which they were involved. The average age of these swimmers was 14 years, and in terms of maturity, they were around the age of peak height velocity. Thus, it is possible that the growth spurt poses stress on the immune system, indirectly supported by Timmons et al. [9], who reported higher resting cytokine levels in boys than in men. This growth-induced stress, in combination with the increased physical stress of athletic training may explain the higher resting TNF- $\alpha$ in the adolescent swimmers. On the other hand, the acute inflammatory response to intense exercise was attenuated in the adolescent swimmers compared with the typical exercise-induced increases we saw in adults, which suggests that single intense bouts of exercise may be immunoprotective, while the chronic accumulation of multiple intense training sessions with little recovery time in between may lead to low-grade inflammation in adolescents. Indeed, the resting levels of both IL- 6 and TNF- $\alpha$ were substantially higher in our group of young swimmers compared with the non-athletic boys in Timmons et al. [9], which could be attributed to the younger age of their group, or to a low-grade inflammation in our swimmers due to intense training. In a previous study, we monitored elite female rowers across a training year, and showed that resting TNF- $\alpha$, as well as IL-1 $\beta$ and IL-6, fluctuated in accordance with training load, with circulating concentrations being highest during periods of high training load and lowest during tapering [29].

Our post-swimming results in the adult group of swimmers are in agreement with previous studies examining cytokine response in men to various forms of exercise, generally showing an increase from pre- to immediately post-exercise regardless of exercise modality [6-9]. Specifically, Zaldivar et al. [7] reported an $80-100 \%$ increase in IL-6 from pre- to immediately post-exercise in young men ( $24.8 \pm 0.8$ years old) performing a 30-minute bout of exercise on a cycle ergometer at $\sim 80 \%$ of their $\mathrm{VO}_{2}$ max. In our study, the young men had a $49 \%$ increase in IL- 6 concentration immediately after the intense swimming trial. However, the study by Zaldivar et al. [7] excluded competitive athletes, so that the exercise regime was novel for participants, which would produce a more robust metabolic stress and immune response. In contrast, our participants were adapted to intense swimming, and chronic exercise training can lead to a blunted cytokine response [30]. In addition, Zaldivar et al. [7] did not adjust for plasma volume changes while a recent study, which did account for plasma volume changes, reported much lower, significant increases in IL-1 $\beta$, IL-6, IL-10 and TNF- $\alpha 5$ min and up to $60 \mathrm{~min}$ after high intensity interval running and cycling in adult men and women [31].

In the adolescent swimmers, our post-swimming results agree with Timmons et al. [9] in that no significant changes occurred in plasma IL- 6 and TNF- $\alpha$ from pre- to post-swimming in this age group. It is important to notice the differences between ours and their study. For example, in the study by Timmons and colleagues [9], participants were non athletes, were of younger age and they 
cycled for $60 \mathrm{~min}$ at a submaximal exercise intensity (i.e., $70 \% \mathrm{VO}_{2 \max }$ ), which is of lower intensity and involves fewer muscle groups than impacted with our swimming protocol. Regardless of these differences, and the fact that they also supplemented participants with a $6 \%$ carbohydrate beverage or placebo, both studies concluded that the inflammatory response to exercise in children and adolescents is lower than what is observed in adults, regardless of training status. According to these authors, children's IL-6 response to sustained, vigorous exercise tends to be approximately $50 \%$ lower than in adults, suggesting that young boys may exhibit a blunted inflammatory response following vigorous exercise [32]. This blunted response seen in boys may be related to their higher resting TNF- $\alpha$ values, suggesting a ceiling effect that requires further research into the causal influences and mechanisms, especially during growth.

The blunted inflammatory response to vigorous exercise in children could also be a protective mechanism during growth. However, we did not measure IGF-1 or other circulating growth factors to support this speculation. A study by Nemet et al. [10] reported significant post-exercise increases in serum IL-1 $\beta$, IL- 6 and TNF- $\alpha$ compared to pre-exercise in adolescent wrestlers (16.5 \pm 0.5 years old) following a standard $1.5 \mathrm{~h}$ wrestling practice. Significant increases from pre- to post-exercise in IL-6 but a decrease in TNF- $\alpha$, were also found in non-athletic adolescent boys and girls ( $14.1 \pm 0.8$ years old) following a stationary bicycle exercise at $\sim 80 \%$ of $\mathrm{VO}_{2 \max }$ [11]. However, it should be noted that this study did not adjust for exercise-induced changes in plasma volume, which may have impacted their results due to effects of hemoconcentration following exercise [33,34]. In addition, our results of a robust increase in IL-10 concentration immediately following high intensity swimming conflict with a previous study in healthy, non-athletic children reporting no changes in IL-10 following $1.5 \mathrm{~h}$ of soccer practice [12]. It is possible, therefore, that the differences in the exercise response among pediatric studies could also be attributed to their differences in mode of exercise employed (swimming, wrestling, cycling, sprinting), as well the relative intensity and duration of the exercise.

In terms of irisin, we observed a small, but significant, decrease in adults with no significant changes in adolescents following the intense swimming trial. These results are difficult to explain. One xplanation could be related to the mode of the exercise being performed. Two previous studies in children and adolescents reported increases in irisin immediately following running and cycling [20,21] while the only other high-intensity swimming study in younger boys and girls found no changes in irisin immediately after each of two repeated $4 \times 50 \mathrm{~m}$ swimming sets [35]. Alternatively, the null findings in our group of boys for irisin may be due to the participants being trained swimmers, and therefore, accustomed to the repeated bouts of swimming. In naïve, adult men, chronic exercise training has been found to result in downregulation of irisin [36], which can also explain the small post-swimming decrease in our adult swimmers, who have been involved with swimming longer. However, previous studies have typically reported increases in irisin levels immediately after different exercise protocols in men $[17,19,21]$. A recent meta-analysis of the effect of acute bouts of exercise on irisin levels in adults found that the overall magnitude of exercise-induced increase in irisin across studies being around 15\% [37]. However, no studies in the meta-analysis included athletes.

The main strength of this study is the consideration of post-exercise plasma volume changes that adds rigour to the experimental design. The interpretation of any post-exercise result is conditional upon establishing whether exercise is effective in eliciting a response beyond the effects of hemoconcentration. A limitation to this current study is its small sample size, which, although similar to that of previous studies on the same topic, may have limited the power to detect significant changes. Another limitation of the study is that the findings are only applicable to the mode of exercise used, i.e., swimming, and may not be applicable to other forms of land-based overground activity such as running. Indeed, it is possible that a land-based activity that is predominantly eccentric and high impact, and as such applies an overall higher stress to the musculoskeletal system, may result to a greater cytokine response across ages. Future studies should consider comparing swimming with equivalent overground land-based running in both male and female athletes for a more complete picture of the inflammatory response to intense exercise. 
In summary, a high intensity interval swimming trial did not induce significant acute increases in inflammatory cytokines, with the exception of anti-inflammatory IL-10, in adolescent male swimmers, while all cytokines, including IL-1 $\beta$, IL-6, IL-10 and TNF- $\alpha$, significantly increased in adult swimmers following the same swimming trial. Likewise, irisin levels did not significantly change in adolescent swimmers, whereas in adult swimmers the levels significantly decreased following high intensity swimming. Our results demonstrate that adolescent athletes display a blunted inflammatory response in contrast to what usually follows exercise in adults. It is possible that training-induced adaptations in adolescents follow a different timescale compared with adults. These results provide a mechanistic insight as to why young athletes require less time to recover from intense exercise and suggest that a different recovery strategy may be effective in this group.

Author Contributions: Conceptualization, P.K., B.J.M. and B.F.; methodology, P.K., B.J.M., A.T., R.K. and B.F.; formal analysis, M.S., A.T., R.K.; investigation, P.K., M.S., B.J.M. and B.F.; resources, P.K. and B.F.; writing-Original draft preparation, M.S.; writing-Review and editing, P.K., B.J.M., R.K. and B.F.; supervision, P.K.; project administration, P.K.; funding acquisition, P.K. All authors have read and agreed to the published version of the manuscript.

Funding: This study was funded by the Natural Sciences and Engineering Research Council of Canada (NSERC grant to P. Klentrou \# 2015-04424). B.J. McKinlay held an Own the Podium-Mitacs Doctoral Scholarship and currently holds an Ontario Graduate Scholarship.

Acknowledgments: We would like to thank all our participants and their parents for participating in our study, graduate and undergraduate students who helped with data collection (Tony Adebero, Nigel Kurgan, Zachary Root, Katherine McKee, Ken Nguyen), as well as the phlebotomists for their assistance.

Conflicts of Interest: The authors declare no conflict of interest.

\section{References}

1. Nieman, D.C.; Wentz, L.M. The compelling link between physical activity and the body's defense system. J. Sport Health Sci. 2019, 8, 201-217. [CrossRef] [PubMed]

2. Suzuki, K. Cytokine Response to Exercise and Its Modulation. Antioxidants 2018, 7, 17. [CrossRef]

3. Bar-Or, O. Pediatric Sports Medicine for the Practitioner: From Physiological Principles to Clinical Applications; Springer: New York, NY, USA, 1983.

4. Falk, B.; Dotan, R. Child-Adult Differences in the Recovery from High-Intensity Exercise. Exerc. Sport Sci. Rev. 2006, 34, 107-112. [CrossRef]

5. Yang, W.; Hu, P. Skeletal muscle regeneration is modulated by inflammation. J. Orthop. Transl. 2018, 13, 25-32. [CrossRef] [PubMed]

6. Ostrowski, K.; Rohde, T.; Asp, S.; Schjerling, P.; Pedersen, B.K. Pro- and anti-inflammatory cytokine balance in strenuous exercise in humans. J. Physiol. 1999, 515, 287-291. [CrossRef]

7. Zaldivar, F.; Wang-Rodriguez, J.; Nemet, D.; Schwindt, C.; Galassetti, P.; Mills, P.J.; Wilson, L.D.; Cooper, D.M. Constitutive Pro- and Anti-Inflammatory Cytokine and Growth Factor Response to Exercise in Leukocytes. J. Appl. Physiol. 2006, 100, 1124-1133. [CrossRef]

8. Zwetsloot, K.A.; John, C.S.; Lawrence, M.M.; Battista, R.A.; Shanely, R.A. High-intensity interval training induces a modest systemic inflammatory response in active, young men. J. Inflamm. Res. 2014, 7, 9-17. [CrossRef]

9. Timmons, B.W.; Tarnopolsky, M.A.; Bar-Or, O. Immune Responses to Strenuous Exercise and Carbohydrate Intake in Boys and Men. Pediatr. Res. 2004, 56, 227-234. [CrossRef] [PubMed]

10. Nemet, D.; Oh, Y.; Kim, H.-S.; Hill, M.; Cooper, D.M. Effect of Intense Exercise on Inflammatory Cytokines and Growth Mediators in Adolescent Boys. Pediatrics 2002, 110, 681-689. [CrossRef]

11. Rosa, J.; Oliver, S.; Flores, R.; Graf, S.; Pontello, A.; Ibardolaza, M.; Zaldivar, F.; Galassetti, P.R. Kinetic Profiles of 18 Systemic Pro- and Anti-Inflammatory Mediators During and Following Exercise in Children. J. Pediatr. Endocrinol. Metab. 2007, 20, 1293-1305. [CrossRef]

12. Scheett, T.P.; Mills, P.J.; Ziegler, M.G.; Stoppani, J.; Cooper, D.M. Effect of Exercise on Cytokines and Growth Mediators in Prepubertal Children. Pediatr. Res. 1999, 46, 429. [CrossRef] [PubMed] 
13. Webber, L.M.; Byrnes, W.C.; Rowland, T.W.; Foster, V.L. Serum Creatine Kinase Activity and Delayed Onset Muscle Soreness in Prepubescent Children: A Preliminary Study. Pediatr. Exerc. Sci. 1989, 1, 351-359. [CrossRef]

14. Soares, J.M.; Mota, P.; Duarte, J.A.; Appell, H.J. Children Are Less Susceptible to Exercise-Induced Muscle Damage than Adults: A Preliminary Investigation. Pediatr. Exerc. Sci. 1996, 8, 361-367. [CrossRef]

15. Kaji, H. Effects of myokines on bone. BoneKEy Rep. 2016, 5, 826. [CrossRef]

16. Mazur-Bialy, A.I.; Pocheć, E.; Zarawski, M. Anti-Inflammatory Properties of Irisin, Mediator of Physical Activity, Are Connected with TLR4/MyD88 Signaling Pathway Activation. Int. J. Mol. Sci. 2017, 18, 701. [CrossRef]

17. Daskalopoulou, S.S.; Cooke, A.B.; Gomez, Y.-H.; Mutter, A.F.; Filippaios, A.; Mesfum, E.T.; Mantzoros, C.S. Plasma irisin levels progressively increase in response to increasing exercise workloads in young, healthy, active subjects. Eur. J. Endocrinol. 2014, 171, 343-352. [CrossRef]

18. Huh, J.Y.; Siopi, A.; Mougios, V.; Park, K.H.; Mantzoros, C.S. Irisin in Response to Exercise in Humans With and Without Metabolic Syndrome. J. Clin. Endocrinol. Metab. 2015, 100, E453-E457. [CrossRef]

19. Kraemer, R.; Shockett, P.; Webb, N.; Shah, U.; Castracane, V. A Transient Elevated Irisin Blood Concentration in Response to Prolonged, Moderate Aerobic Exercise in Young Men and Women. Horm. Metab. Res. 2013, 46, 150-154. [CrossRef]

20. Löffler, D.; Müller, U.; Scheuermann, K.; Friebe, D.; Gesing, J.; Bielitz, J.; Erbs, S.; Landgraf, K.; Wagner, I.V.; Kiess, W.; et al. Serum Irisin Levels Are Regulated by Acute Strenuous Exercise. J. Clin. Endocrinol. Metab. 2015, 100, 1289-1299. [CrossRef]

21. Huh, J.Y.; Mougios, V.; Kabasakalis, A.; Fatouros, I.; Siopi, A.; Douroudos, I.I.; Filippaios, A.; Panagiotou, G.; Park, K.H.; Mantzoros, C.S. Exercise-Induced Irisin Secretion Is Independent of Age or Fitness Level and Increased Irisin May Directly Modulate Muscle Metabolism Through AMPK Activation. J. Clin. Endocrinol. Metab. 2014, 99, E2154-E2161. [CrossRef]

22. Hirose, L.; Nosaka, K.; Newton, M.; Laveder, A.; Kano, M.; Peake, J.; Suzuki, K. Changes in inflammatory mediators following eccentric exercise of the elbow flexors. Exerc. Immunol. Rev. 2004, 10, 75-90. [PubMed]

23. Nagle, E.F.; Sanders, M.E.; Franklin, B.A. Aquatic High Intensity Interval Training for Cardiometabolic Health. Am. J. Lifestyle Med. 2016, 11, 64-76. [CrossRef] [PubMed]

24. Espersen, G.T.; Elbaek, A.; Schmidt-Olsen, S.; Ejlersen, E.; Varming, K.; Grunnet, N. Short-term changes in the immune system of elite swimmers under competition conditions: Different immunomodulation induced by various types of sport. Scand. J. Med. Sci. Sports 2007, 6, 156-163. [CrossRef] [PubMed]

25. Peake, J.M.; Neubauer, O.; Della Gatta, P.A.; Nosaka, K. Muscle damage and inflammation during recovery from exercise. J. Appl. Physiol. 2017, 122, 559-570. [CrossRef] [PubMed]

26. McKinlay, B.J.; Theocharidis, A.; Adebero, T.; Kurgan, N.; Fajardo, V.A.; Roy, B.D.; Josse, A.R.; Logan-Sprenger, H.M.; Falk, B.; Klentrou, P. Effects of Post-Exercise Whey Protein Consumption on Recovery Indices in Adolescent Swimmers. Int. J. Environ. Res. Public Health 2020, 17, 7761. [CrossRef]

27. Mirwald, R.L.; Baxter-Jones, A.D.G.; Bailey, D.A.; Beunen, G.P. An assessment of maturity from anthropometric measurements. Med. Sci. Sports Exerc. 2002, 34, 689-694. [CrossRef]

28. Van Beaumont, W. Evaluation of hemoconcentration from hematocrit measurements. J. Appl. Physiol. 1972, 32, 712-713. [CrossRef]

29. Kurgan, N.; Logan-Sprenger, H.; Falk, B.; Klentrou, N. Training, Inflammation and Bone Markers in Elite Female Rowers during an Olympic Year. Med. Sci. Sports Exerc. 2018, 50, 1810-1817. [CrossRef]

30. Gokhale, R.; Chandrashekara, S.; VasanthaKumar, K. Cytokine response to strenuous exercise in athletes and non-athletes-An adaptive response. Cytokine 2007, 40, 123-127. [CrossRef]

31. Kouvelioti, R.; Kurgan, N.; Falk, B.; Ward, W.E.; Josse, A.R.; Klentrou, P. Cytokine and Sclerostin Response to High-Intensity Interval Running versus Cycling. Med. Sci. Sports Exerc. 2019, 51, 2458-2464. [CrossRef]

32. Timmons, B.W. Exercise and Immune Function in Children. Am. J. Lifestyle Med. 2007, 1, 59-66. [CrossRef]

33. Kargotich, S.; Goodman, C.; Keast, D.; Morton, A.R. The Influence of Exercise-Induced Plasma Volume Changes on the Interpretation of Biochemical Parameters Used for Monitoring Exercise, Training and Sport. Sports Med. 1998, 26, 101-117. [CrossRef] [PubMed]

34. Weinstein, Y.; Bediz, C.; Dotan, R.; Falk, B. Reliability of peak-lactate, heart rate, and plasma volume following the Wingate test. Med. Sci. Sports Exerc. 1998, 30, 1456-1460. [CrossRef] [PubMed] 
35. Kabasakalis, A.; Nikolaidis, S.; Tsalis, G.; Christoulas, K.; Mougios, V. Effects of sprint interval exercise dose and sex on circulating irisin and redox status markers in adolescent swimmers. J. Sports Sci. 2019, 37, 827-832. [CrossRef] [PubMed]

36. Norheim, F.; Langleite, T.M.; Hjorth, M.; Holen, T.; Kielland, A.; Stadheim, H.K.; Gulseth, H.L.; Birkeland, K.I.; Jensen, J.; Drevon, C.A. The effects of acute and chronic exercise on PGC- $1 \alpha$, irisin and browning of subcutaneous adipose tissue in humans. FEBS J. 2013, 281, 739-749. [CrossRef]

37. Fox, J.; Rioux, B.V.; Goulet, E.D.; Johanssen, N.M.; Swift, D.L.; Bouchard, D.R.; Loewen, H.; Sénéchal, M. Effect of an acute exercise bout on immediate post-exercise irisin concentration in adults: A meta-analysis. Scand. J. Med. Sci. Sports 2017, 28, 16-28. [CrossRef]

Publisher's Note: MDPI stays neutral with regard to jurisdictional claims in published maps and institutional affiliations.

(C) 2020 by the authors. Licensee MDPI, Basel, Switzerland. This article is an open access article distributed under the terms and conditions of the Creative Commons Attribution (CC BY) license (http://creativecommons.org/licenses/by/4.0/). 